\title{
Phronesis
}

\section{Certitudes et hésitations des institutions scolaires françaises entre instruction, socialisation et qualification. Un point de vue historique sur la longue durée Certainties and doubts of French educational institutions between education, socialization and qualification. An historical point of view on a long time period}

\author{
André D. Robert
}

Volume 2, numéro 2-3, avril-juillet 2013

Intervention et pratique éducative : Reflet et/ou tensions entre instruction, socialisation et qualifications?

URI : https://id.erudit.org/iderudit/1018078ar

DOI : https://doi.org/10.7202/1018078ar

Aller au sommaire du numéro

Éditeur(s)

Institut de recherche sur les pratiques éducatives

ISSN

1925-4873 (numérique)

Découvrir la revue

Citer cet article

Robert, A. D. (2013). Certitudes et hésitations des institutions scolaires françaises entre instruction, socialisation et qualification. Un point de vue historique sur la longue durée. Phronesis, 2(2-3), 105-113.

https://doi.org/10.7202/1018078ar

\section{Résumé de l'article}

Revendiquant l'héritage des révolutionnaires de 1789, les républicains qui organisent à la fin du XIXe siècle l'école française dans sa forme moderne développent une philosophie qui se veut " fondationnaire», plaçant l'institution scolaire au fondement de la nation. L'idée d'une émancipation du peuple par l'instruction rationnelle sans négliger l'éducation morale et civique est ainsi située au coeur du projet scolaire français et vise des sujets rationnels abstraits, nonobstant leurs caractéristiques et appartenances singulières. La formule « la République sera éducatrice ou elle ne sera pas ! » affirme cette intention fondamentalement politique qui 'tient' grosso modo jusqu'à la seconde guerre mondiale. A l'issue de celle-ci, l'institution scolaire commence à être saisie en profondeur par l'économie ; pendant la période de

l'Etat-Providence, une synthèse originale paraît s'opérer entre « la méritocratie républicaine, [...] l'égalité sociale de masse ... [le] souci individualiste " (Gauchet, 2002), les préoccupations montantes de l'économie et de l'emploi, faisant de la question des qualifications une préoccupation de plus en plus centrale. Cet équilibre fragile semble ensuite se défaire pendant les Vingt Piteuses, l'école se caractérisant alors par une démultiplication de ses missions et une perte de repères, une difficulté à faire sens. C'est la signification présente de l'institution scolaire qui sera interrogée, au regard de l'influence économico-anthropologique du néo-libéralisme, de l'exigence d'une formation 'tout au long de la vie' et ses conséquences, ainsi que - par le fait - de la dialectique instruction-socialisation-qualification. 


\section{Certitudes et hésitations des institutions scolaires françaises entre instruction, sociali- sation et qualification. Un point de vue historique sur la longue durée}

\section{André D. ROBERT}

Université Lyon 2 (France)

Laboratoire Education, Cultures et Politiques (EA 3571).

86 Rue Pasteur

69007 Lyon

andre.robert@univ-lyon2.fr

Mots-clés : France, institutions scolaires, instruction, qualification, socialisation, histoire du système éducatif.

Résumé: Revendiquant l'héritage des révolutionnaires de 1789, les républicains qui organisent à la fin du XIXe siècle lécole française dans sa forme moderne développent une philosophie qui se veut "fondationnaire», plaçant l'institution scolaire au fondement de la nation. L'idée d’une émancipation du peuple par l'instruction rationnelle sans négliger léducation morale et civique est ainsi située au coeur du projet scolaire français et vise des sujets rationnels abstraits, nonobstant leurs caractéristiques et appartenances singulières. La formule "la République sera éducatrice ou elle ne sera pas! » affirme cette intention fondamentalement politique qui 'tient' grosso modo jusqu’à la seconde guerre mondiale. A l'issue de celle-ci, l'institution scolaire commence à être saisie en profondeur par léconomie; pendant la période de l'Etat-Providence, une synthèse originale paraît sopérer entre "la méritocratie républicaine, [...] légalité sociale de masse ... [le] souci individualiste » (Gauchet, 2002), les préoccupations montantes de léconomie et de lemploi, faisant de la question des qualifications une préoccupation de plus en plus centrale. Cet équilibre fragile semble ensuite se défaire pendant les Vingt Piteuses, lécole se caractérisant alors par une démultiplication de ses missions et une perte de repères, une difficulté à faire sens. C'est la signification présente de l'institution scolaire qui sera interrogée, au regard de l'influence économico-anthropologique du néo-libéralisme, de lexigence d’une formation 'tout au long de la vie' et ses conséquences, ainsi que - par le fait de la dialectique instruction-socialisation-qualification.

\section{Title: Certainties and doubts of French educational institutions between education, socialization and qualification. An historical point of view on a long time period}

Keywords: France, educational institutions, education, skills, socialization, history of education

\footnotetext{
Abstract: Claiming the legacy of revolutionary of 1789, the Republicans organise at the end of nineteenth century the French school in its modern form and developpe a philosophy that is "fondationnaire" placing educational institution as foundation of the nation. The idea of the emancipation of the people by rational instruction without neglecting the moral and civic education is well located in the heart of the French school project and aims abstract rational subjects, despite their unique characteristics and affiliations. The phrase "the Republic will be educator or she will not! "claim this fundamentally political intent stay up until the Second World War. After this period, the school system begin to be invested by the economy; during the period of the welfare state, an original synthesis seems to occur between "republican meritocracy, [...] social equality mass ... [the] concern individualistic "(Gauchet, 2002), the rising concerns of the economy and employment, making the question of the qualifications of a concern more central. This delicate balance then seems to break during the Twenty pitiful's years, then the school is characterized by a multiplicating of its missions, the lost of landmarks and the difficulty to make sense. The actual siginification of the scholar system will be questioned regarding to economic and anthropological influence of neo-liberalism, the requirement for long life education and its consequences, and also the education-socialization-qualification relations.
} 


\section{Introduction}

Afin de tracer les contours problématiques d'une contribution centrée autour des trois termes que toute intention scolaire met nécessairement en tension (instruction, socialisation, qualification), je voudrais placer en regard l'un de l'autre - dans le cadre constitué par les politiques et les finalités scolaires en France - les propos de deux personnalités intellectuelles très différentes, ne serait-ce d’abord que par leur appartenance générationnelle, le professeur de grec Maurice Lacroix d’une part, la sociologue Lucie Tanguy d’autre part. La référence à ces deux personnes est une manière d'entrer dans la considération de la longue durée, puisque seront concernées ici aussi bien l'histoire de l'institution scolaire française de la fin du XIX et de la première moitié du XXe siècle que son histoire contemporaine.

Maurice Lacroix (1893-1989), agrégé de lettres en 1919, a enseigné dans plusieurs lycées et terminé sa carrière en occupant la chaire de grec en classe de Première Supérieure au lycée Henri IV ; il incarne ainsi lélite professorale de l'enseignement secondaire français, ce "puissant Empire du milieu » (selon l'expression de l'historien Lucien Febvre). Parallèlement à sa carrière de professeur, il a mené une activité militante dans plusieurs organisations syndicales, politiques et religieuses, catholiques. En 1937, il est l'un des fondateurs du Syndicat des professeurs de l'enseignement secondaire, organisation restée très influente chez les professeurs français du second degré. Ses engagements syndicaux et politiques le situent clairement à gauche. Lors de son décès, l'historien Jean-Pierre Rioux lui a rendu hommage en ces termes : "Ce chrétien fervent, qui joua un grand rôle à la paroisse universitaire, avait puisé dans l'amitié et l'exemple de Marc Sangnier la force de ses choix : un engagement laïque sans concessions, une fierté républicaine à toute épreuve, l'espoir d'un socialisme à visage humain, une fidélité à soi-même et à son métier »1 (cité par Seguy, 2010, p. 195). Sa personnalité nous intéresse pour toutes ces raisons, et en l'occurrence particulièrement pour celle-ci : ardent défenseur d'une certaine conception de l'enseignement secondaire et des humanités, tout en étant favorable à la démocratisation scolaire, il sest fortement opposé à plusieurs réformes impliquant une éventuelle redistribution des relations instruction, socialisation, qualification, en ne pouvant imaginer autre chose que l'exclusivité donnée à l'instruction2, c'est-à-dire l'action de communiquer des connaissances et de former l'esprit d'un enfant.

Lucie Tanguy, directrice de recherche au CNRS, n’a pas besoin dêtre présentée, ses travaux de sociologie du travail et de la formation sont en effet bien connus dans la francophonie (par exemple : L'introuvable relation formation-emploi, L'enseignement professionnel en France, et tout récemment : Une histoire de la sociologie du travail en France 1950-1990). Au centre de son œuvre sociologique se trouve ce qu'lle appelle « le basculement de l’ordre scolaire » désigné par le glissement de la notion d'éducation à celle de formation, problème qui recouvre le champ de nos interrogations présentes. A l'occasion d'un récent jury de soutenance d'habilitation à diriger des recherches, elle s'exprimait en ces termes : « Loin dêtre un simple glissement sémantique, [ce glissement] indique des changements politiques majeurs, qui ont placé les préoccupations de l'emploi au cœur du système éducatif : politiques éducatives qui sont désormais énoncées en termes de niveau de formation ou de qualification, formules qui témoignent de cette volonté, toujours réaffirmée, d’établir des relations d'équivalence entre ces quatre registres différents de la réalité sociale que sont léducation, la formation, la qualification et l'emploi occultant ainsi que la formation n’est qu’une composante de la qualification et que celle-ci se définit sur le marché du travail et au sein des entreprises. C'est dans le cadre du Plan (des années 1960) que des politiques et des experts économiques ont fait admettre sur la scène publique la nécessité de lier l'éducation à l'économie, ont promu la notion de formation, en lieu et place de l'éducation, ont fait apparaître cette notion comme une grandeur mesurable et l'ont posée au fondement de la qualification $»^{3}$.

Quant à Maurice Lacroix, dont on se rappellera l'ensemble des caractéristiques, voici comment il sexprimait il y a un peu plus de cinquante ans, alors même que le mouvement de modernisation de lécole sétait amorcé sous la Quatrième et allait bientôt s’accélérer dans les années suivantes sous le nouveau régime de la Cinquième République : « Il est, sans doute, désirable que l’enfant ne soit pas jeté dans la vie sans avoir acquis certaines connaissances dont l'homme aura besoin pour l'exercice de son métier. Il est encore plus nécessaire qu'il soit préparé à sa profession d'homme, quelle que soit la spécialité dans laquelle son activité se déploiera. (...) Mais, en fait, on s'oriente de plus en plus vers un

1 Journal Le Monde, «La mort de M. Lacroix, la vaillance du 'Croux’ », 22.02.1989.

2 Du verbe latin instruere : assembler, bâtir, équiper.

3 Rapport de soutenance de l'habilitation à diriger des recherches de Henri Eckert, EHESS Paris, 1.12.2009, non publié. 
système de préparation indirecte : on tend à organiser les diverses branches de l'enseignement en fonction des débouchés, donc à substituer à la notion de culture celle de préparation préprofessionnelle. Nous devons dire notre désaccord. Le métier n'est pas toute la vie de l'homme. Il faut assurer à l'enfant, dans la mesure de ses aptitudes, une culture générale aussi large que possible. Nécessaire pour l’ensemble de son activité humaine et civique, elle ne lui sera pas moins utile dans la vie professionnelle en un temps où les conditions du marché du travail évoluent rapidement et où le problème de reconversion peut se poser avec acuité »4 (cité par Seguy, 2010, p. 541-542).

Sur deux registres de langue bien différents, l’un militant (Maurice Lacroix), l'autre analytique et scientifique (Lucie Tanguy), nous avons affaire avec ces propos à l'agencement de questionnements implicites qui permettent d’esquisser une mise en perspective problématique de l'histoire du système éducatif français. A un pôle, historiquement le plus lointain, nous pourrions penser trouver, conformément à la conviction du professeur de grec, une prédominance de l'instruction - comme prétend le traduire le nom du ministère de l'Instruction publique (en vigueur des années 1880 jusquen 1932, date à laquelle seulement il devient ministère de l'Education nationale) - mais la réalité historique nous fait rencontrer une forte attention portée à l'éducation morale, à l'inculcation de valeurs et d'habitus, c'est-à-dire à une forme de socialisation par laquelle " une société se dote d'acteurs capables d’assurer son intégration » (Dubet, Martuccelli, 1996). Notons qu'un autre aspect de la socialisation ainsi comprise (socialisation républicaine et démocratique, pourrait-on dire) vise par ailleurs à doter la société « d'individus, de sujets, susceptibles de produire une action autonome »5. En France, nous voyons se stabiliser pour un temps dans l'institution scolaire un couple instruction-éducation, dont la modulation varie selon le type d’enseignement (pour les élèves du primaire, priorité donnée à léducation, c'est-à-dire à la socialisation intégrative, sans négliger une instruction de base ; pour les élèves du secondaire, priorité effectivement donnée à l'instruction, c'est-à-dire plutôt à la socialisation autonomisatrice, du moins sur le plan intellectuel, étant entendu qu'elle se combine avec une distinction éducative transmise familialement). A l'autre pôle, qui caractérise l'époque contemporaine (depuis la deuxième moitié du vingtième siècle), nous assistons, conformément à l'analyse de la sociologue, à une redistribution des rapports entre léducation (couplée à l'instruction), la formation et la qualification au bénéfice quasi exclusif d'une conception de plus en plus économiste de celle-ci (étroitement tributaire du marché de lemploi). Cette redistribution, dont la logique, accordée aux évolutions du monde contemporain, paraît certes incontournable (comme en atteste l'actuelle formulation québécoise des missions du système éducatif), pose cependant la question de la position respective des termes, et surtout des réalités qu'ils recouvrent, au sein de la trilogie. La domination, voire l'exclusivité, de l'obsession qualificative ne manque pas d'interroger le sens conféré à la volonté de scolariser, et engage en dernière analyse une philosophie de l'éducation - toutes questions qui ne peuvent être déconnectées de la prise en compte d'un contexte historico-national.

Comment se sont opérés historiquement en France les glissements d'un pôle à l'autre, grossièrement définis au niveau de cette introduction ? Dans l’optique d'une philosophie humaniste, ancrée dans la tradition républicaine à la française, quel niveau de compromis peut-il être considéré comme acceptable entre instruction, socialisation et qualification ? Je distinguerai, dans une perspective très cavalière, trois grandes périodes, correspondant à des postures de l'Etat central relativement aux fonctions assignées à l'institution scolaire, eu égard à ces trois notions et leurs corollaires, en me situant nécessairement d’un point de vue « macro ».

1. Première période : Etat éducateur ou enseignant, Ecole conquérante, sûre d'elle-même, mais séparatrice (18801940)

Qualifier l'Etat d'éducateur ou d'enseignant autour de la mise en œuvre des grandes lois scolaires républicaines françaises renvoie directement à Jules Ferry lorsqu'il déclare devant le Sénat le 5 mars $1880:$ : Il y a deux choses dans lesquelles l'Etat enseignant et surveillant ne peut pas être indifférent ; c'est la morale et la politique, car en morale, comme en politique, l'Etat est chez lui ; c'est son domaine et par conséquent sa responsabilité ». Alors que les contenus d'enseignement vont être contrôlés par l'appareil d'Etat pour la première fois sans référence explicite à l'Eglise, et concerner tous les enfants français, la mission d'instruction primaire étant confiée à lécole publique (article 4 de la loi du 28 mars 1882), le curriculum - tel qu'il s'expose dans l'article premier de la même loi relative à l'enseignement primaire - place en tête l'instruction morale et civique, devant toutes les autres composantes (constitués de savoirs et savoir-faire plus objectifs, certains directement orientés vers la pratique).

On n'insistera pas sur le fait que, quand bien même il ne se donnerait pas d’objectif d'instruction portant sur des savoirs dûment repérables, tout processus déducation comprend, dans son déploiement, une forme de socialisation entendue comme inculcation de " manières d’agir, de penser et de sentir ", assurant une mise en forme sociale des dispositions présentes en l'individu appelé à stabiliser progressivement son appartenance à une société et à certains de ses groupes intermédiaires, tout en contribuant à la cohésion de cette société. Quoique désignées

4 «Face au péril », Revue de la franco-ancienne, avril 1958.

Ibid. Concernant le premier mouvement du processus, E. Durkheim a largement souligné l'importance primordiale de l'école dans la formation morale du pays. 
ici comme objets d'une instruction, la morale et les valeurs civiques qui constituent la priorité du programme primaire républicain relèvent d'une socialisation ainsi conçue, en ce sens que la mission assignée à l'Ecole consiste à faire éprouver de la manière la plus aiguë le sentiment d’appartenance à la Nation. Comme le dit explicitement dans une réunion publique Eugène Spuller, plusieurs fois ministre, à qui lon doit la formule « La République sera éducatrice ou elle ne sera pas » : « C'est beaucoup que de rendre à leurs familles de petits écoliers bien instruits et bien cultivés, mais il faut encore en faire des hommes pour la France, des citoyens pour la République [...] Où se fera cette unité morale dont nous avons tant besoin, après tant de ruines, tant de désastres, de discordes et de malheurs ? Messieurs, elle ne peut se faire quà l'école, où l'on vit dans la sphère des idées les plus désintéressées et les plus élevées » (Spuller, 1892). Se réclamant de l'héritage des révolutionnaires de 1789 et de certains de ceux qui les ont suivis (1848), les Républicains, tel précisément Eugène Spuller, revendiquent une philosophie qui se veut "fondationnaire", c'est-à-dire situant lécole au fondement de la construction nationale. Placée sous l'autorité de valeurs morales et civiques à vaste portée, puisque déclarées universelles et concernant l'humanité tout entière, lécole est assignée à la mission prioritaire denseigner ces valeurs et soumise à la responsabilité - dans ce même mouvement - d'instituer la nation en la personne des élèves futurs citoyens. Il nest point besoin de recourir à la notion de curriculum caché pour rendre compte de la finalité ainsi dévolue à l'enseignement primaire tant son contenu explicite est affiché et revendiqué, à côté ou même confondu avec les objectifs d'instruction. L'idée d'une émancipation du peuple par 1) léducation morale et civique, 2) une instruction rationnelle (quoique limitée à « ce qu'il n’est pas permis d'ignorer ») se trouve au cœur du projet scolaire français de masse, visant des sujets abstraits, en principe sans considération de leurs caractéristiques et appartenances singulières. Ainsi l'équation sur laquelle repose l'institution scolaire française à ses origines est de nature essentiellement politique (ce qui ne veut pas dire - comme on va le voir - quelle nocculte pas d'autres fonctions, à caractère économique et social), et l'on peut suivre alors l'analyse de Bernard Charlot :

L'action de l'Etat [au cours de cette période, la Troisième République] est politique, philosophique, morale, culturelle et non pas économique. Ou plus exactement, l'Etat éducateur ne remplit une fonction économique que de façon indirecte : par l'éducation, il pacifie la société et assure ainsi l'ordre nécessaire à la prospérité économique de la bourgeoisie »(Charlot, 1994, p. 28).

Da fait, contradictions, tensions, inégalités ne manquent pas d’apparaître dès lorigine, même si elles sont théorisées-justifiées par les promoteurs du projet républicain et ne font qu'exceptionnellement l'objet de critiques, mais virulentes et parfois formulées du sein même de l'institution (par exemple par Ferdinand Buisson, le célèbre auteur du Dictionnaire de pédagogie). En effet, sûre d’elle-même, conquérante, lécole de l'Etat éducateur est en même temps foncièrement séparatrice. Ainsi l'enseignement secondaire d'Etat est payant (il le restera jusqu’en 1930) ; tout en se disant seulement préoccupé d'instruction à l'intention des élèves « capables », il est caractérisé par une distinction culturelle longtemps concentrée autour de l'enseignement du latin et des humanités, il englobe un enseignement élémentaire dès les petites classes avant la 6e, parallèle de l'enseignement primaire et réservé à une minorité sociale. Cela conduit le philosophe Edmond Goblot à recourir à l'image de la «barrière " pour évoquer la manière dont la bourgeoisie entend se protéger du peuple - cette barrière étant essentiellement constituée par le latin (Goblot, 1925, rééd. 2010). Cet enseignement ne saurait avoir aucune finalité professionnelle, le rattachant en quoi que ce soit à une notion de qualification, il prend le temps long d'une socialisation culturelle dans l'enseignement secondaire (Robert, 2005). La question de la formation professionnelle est en partie renvoyée, dans sa forme scolaire, aux Ecoles primaires supérieures ou « collèges du peuple » (Briand, Chapoulie, 1992). Des contre-projets, des tentatives de réforme et quelques réformes (particulièrement sous le Front Populaire, 1936-1939), de timides avancées de la démocratisation, des mesures de correction des traits les plus négatifs de cette organisation scolaire traversent la période sans parvenir à modifier en profondeur l'institution.

Pour conclure cette présentation nécessairement très elliptique de la première période, je voudrais marrêter sur une posture idéologique, que j’ai repérée chez une catégorie enseignante particulière, les professeurs des classes élémentaires de lycée (classes avant la 6e précisément), posture promise à une certaine postérité. Il s’agit de ce que j’ai appelé « démo-élitisme ». Par cet oxymore, j’entends une attitude idéologique associant un souci de démocratisation de l'enseignement (qui accepte délargir les bases sociales du recrutement secondaire en fonction des aptitudes) à une défense militante de la culture secondaire traditionnelle. Tandis que la notion délitisme républicain vise à dégager une élite de lécole du peuple au sein d'un continuum premier/second degré, le démo-élitisme pose d’emblée et a priori l'autonomie, la supériorité et la distinction définitive du secondaire. A ce titre, prétendant ne vouloir reconnaitre à son fondement que l'instruction (l'instruction la plus haute) il incarne la socialisation distinctive de l'enseignement secondaire, abandonnant toute préoccupation même très indirecte de qualification au secteur de l'enseignement primaire ou surtout professionnel, comme le souligne le texte de Maurice Lacroix cité dans notre introduction. 


\section{Deuxième période : Etat-Providence modernisateur, Ecole saisie par l'économie, démocratisation rela- tive (1945-1975)}

Sans bien sûr que la période antérieure ait été exempte d’interrogations économiques et sociales explicites relatives à la fonction de lécole dans la société française (mais surtout concentrées sur les secteurs des enseignements professionnels et techniques, sur celui dit du «primaire supérieur »), les conséquences de la seconde Guerre Mondiale, les nécessités de la reconstruction et les nouvelles conditions de la compétition économique internationale constituent un tournant quant à la mutation de cette fonction, et quant à lémergence significative de la notion de formation -ainsi que des termes de la même série paradigmatique, comme qualification - au cœur des préoccupations scolaires, principalement de second degré.

Dans l'immédiat Après-Guerre, l'institution scolaire est saisie par léconomie. A partir du milieu des années cinquante, parallèlement à une forte demande sociale d'enseignement secondaire sensible dans de nombreux pays, l'enseignement devient mondialement (du moins dans la sphère des nations développées) un objet d'investigation économique6. La théorie économique dite du " capital humain » sempare de la question scolaire. Examinant le rythme de développement de divers pays dans l'histoire économique longue, certains chercheurs ont été amenés à constater que le capital physique n’a pas un rôle aussi déterminant dans la croissance économique quon avait pu le penser auparavant et que les trois facteurs de production fréquemment mentionnés (terre, capital, travail) ne suffisent plus à expliquer l'accroissement du produit national, compte tenu d'hypothèses comme celle des rendements dits décroissants, c'est-à-dire dont la rentabilité n’est ni maximale ni immédiate. Des économistes inventent la notion de «facteur résiduel », quatrième facteur qui est censé englober le progrès technique, laccroissement des connaissances, la qualification de la force de travail, le niveau général d'instruction de la population. Peu à peu, la majeure partie sinon la totalité de ce facteur résiduel se voit dévolue à l'enseignement, en raison du fait que l'institution scolaire a spécifiquement pour mission d'accumuler et de transmettre les connaissances qui sont les conditions du progrès technique, un des tout premiers éléments à lorigine de la croissance.

Tel est le cas de la France. Voulu modernisateur et développeur, enrôlant désormais explicitement lécole dans le processus économique au nom du capital humain, l'Etat se fait planificateur. Le commissariat général au Plan est créé en 1946 ; son promoteur-théoricien Jean Monnet présente en 1947, dans le contexte législatif de la Quatrième République, le premier Plan de modernisation et d’équipement, destiné à indiquer tous les quatre ou cinq ans au gouvernement les évolutions économiques probables comme les grandes lignes des mesures à prendre, notamment en matière de formation. Dans la même période, sont établies dans le secteur de lemploi salarié (par les arrêtés dits Parodi de 1946), des grilles consistant en des nomenclatures hiérarchisées d’emploi fondées sur une correspondance entre savoir-faire (donc qualification), emploi occupé et salaire minimum ; il est clair qu’en amont de ces instruments de classement, revendiqués et négociés par les organisations syndicales de travailleurs, se trouvait posée la question cruciale du type de formation à acquérir. Le général De Gaulle est entièrement rallié à cette conception planificatrice, ce qui le conduit à soutenir l'idée, tant en matière économique que scolaire, de " compenser l'inconvénient de la liberté sans en perdre l'avantage » (De Gaulle, 1970, p. 143), contribuant ainsi à soutenir une économie capitaliste régulée et à prétendre mettre à son service un système éducatif lui-même fortement organisé. Il s’agit de faire le plus possible coïncider aptitudes puisées dans tous les milieux sociaux, niveaux différenciés de qualification et besoins de cette économie. La question des niveaux (de formation et de qualification) commence à supplanter dans l'Ecole celle de la «barrière ».

De fait, les mesures de réforme de l'institution scolaire française s'inscrivent à partir de 1959, et pendant la période faste dite des « Trente Glorieuses » (1945-1975), dans cette perspective, les premières consistant à prolonger la scolarité obligatoire jusqu'à seize ans et à prétendre ouvrir à tous les petits français les rudiments d'une scolarité désormais appelée " de second degré ». Il s’agit d’élargir très considérablement le vivier des jeunes susceptibles de recevoir une formation initiale de second degré (à défaut d'une socialisation secondaire au sens traditionnel, revendiquée par les partisans des humanités du type Maurice Lacroix). Cet accroissement du nombre des bénéficiaires d'un début d'enseignement du second degré relève plus des besoins nouveaux de l'économie que de la bonne volonté des gouvernants, mais cela va malgré tout dans la direction d'une démocratisation quantitative. Autre signe du déplacement de la problématique de la formation (et partant de la qualification) de la périphérie au centre du système éducatif : l'enseignement professionnel, qui relevait d'un secteur propre, est désormais intégré à l'ensemble éducatif organisé en degrés.

Le propos n’est pas ici de détailler les difficultés et les contradictions suscitées par ces réformes, ni lenchaînement des autres mesures qu’elles ont entraînées tant par le constat des échecs dont elles étaient porteuses que sous l'effet de luttes sociales menées au nom de la démocratisation. Les politiques scolaires menées pendant les « Trente Glorieuses » sous l'égide de l'Etat-Providence modernisateur sont certes traversées

$6 \quad$ Tout le développement qui suit s’inspire de Segré, Tanguy, (1970). 
par toutes les contradictions inhérentes au système capitaliste fondé sur la domination d'une classe sur toutes les autres, comme y insiste dès le milieu des années 1960 le courant de la sociologie critique. Le mouvement de rénovation des contenus d'enseignement mettant en cause l'ancienne organisation du secondaire entraîne de fortes résistances, qui transcendent le clivage gauche/droite. Les tenants des humanités et de la barrière séparatrice voient se défaire l'ordre secondaire ancien, et le déplorent. A gauche, l’attitude démo-élitiste trouve à se redéployer sous l'idée d'une acceptation de lélargissement des bases sociales de lenseignement du second degré mais en maintenant la perpétuation de la tradition pédagogique secondaire au nom du haut niveau d'exigence auquel ont droit tous les élèves - ce qui va bloquer durablement toute rénovation pédagogique en profondeur. On peut parler d'une volonté de maintien de la socialisation traditionnelle propre à l'enseignement secondaire alors même que les conditions et finalités de l'enseignement et de l'instruction ont commencé à profondément changer.

Il demeure que cette période, marquée par l'alliance des économistes et des réformateurs scolaires (1945-1975) se caractérise par une progression spectaculaire en matière de démocratisation de la fréquentation d'un enseignement de second degré : + 438\% en trente ans, cela ne préjugeant pas de la nature de la démocratisation qualitative. Que cette politique ait plus été dictée par des motivations économiques en rapport avec les nouveaux besoins du marché du travail que par une forme de générosité sociale importe peu si, au total et très globalement, idéaux de justice et finalités matérialistes ont tendu à se rapprocher. On peut risquer l'idée d'un équilibre précaire alors réalisé, dans une école restant encore assurée d’elle-même nonobstant ses défaillances et les critiques dont elle est lobjet, entre instruction, socialisation, formation et qualification. Le philosophe Marcel Gauchet propose sa vision de léquilibre en question : « L'école de l'Etat-Providence opère la synthèse - dont on ne discerne pas à lépoque ce qu’elle a de provisoire - de la méritocratie républicaine, de l'égalité sociale de masse et du souci individualiste. Elle poursuit en effet un triple objectif. Elle entend assurer simultanément légalité des chances, avec ce que cela suppose de correction des situations de départ et donc de tension sélective, et l’ouverture à tous de la meilleure éducation possible, tout en dispensant dans ce cadre une éducation individuelle et libre. Et, ô miracle, dans une large mesure, elle y parvient » (Gauchet in Blais, Gauchet, Ottavi, 2002, p. 33).

\section{Troisième période : Etat managérial et néolibéralisme, Ecole incertaine, dangers (1980-2010)}

A la charnière entre deuxième et troisième périodes, à la faveur notamment des crises politiques et économiques, lécole devient plus incertaine d’elle-même. La sociologie a pu décrire ce fait en analysant la multiplication des principes de justice se déployant au sein de l'institution scolaire, le principe civique - fondateur et unique au cours de la le période - gardant une place, tout en étant attaqué et ne jouissant plus du monopole au bénéfice d'autres principes concurrents, industriel, marchand, domestique, artiste, parfois se combinant entre eux (Derouet, 1992). Au terme des alternances politiques gauche/droite survenues au cours de cette 3e période, on se trouve en France face à un extraordinaire empilement de mesures successives (réformes continuelles du primaire, du collège, du lycée, plans de lutte contre la violence, contre l'illettrisme, multiplication des Hauts Conseils) auxquelles chaque ministre veut attacher son nom, mais qui ne parviennent pas à faire complètement sens (Gauthier, 2011). Apparaît une confusion des missions de l'école, difficiles désormais à ordonner et hiérarchiser, du fait de la multiplication des injonctions qui lui sont assignées par la sphère politique et par la société elle-même : donner des chances égales à tous, placer l'élève au centre du système et permettre son épanouissement, instruire, éduquer, socialiser, cultiver, répondre aux défis économiques du monde contemporain dans un contexte de concurrence exacerbée, élever le niveau général de la population, préparer à l'emploi et professionnaliser, sélectionner les élites, contribuer à la lutte contre l'exclusion, réduire la violence, compenser certaines défaillances parentales et pour tout dire apporter des remèdes aux maux sociaux que d'autres institutions n'arrivent pas à guérir, etc.

La création en 1985 du baccalauréat professionnel constitue un indice révélateur des incertitudes désormais structurelles dans lesquelles sest installée l'institution scolaire. Cette création s'inscrit dans le cadre d'une politique, cette fois menée par la gauche, qui entend poursuivre et renouveler (de meilleure façon que celles qui lont précédée) l'alliance entre l'école et l'économie, la formation et la qualification, l'instruction et la socialisation. L`exemple japonais est alors souvent invoqué à titre de modèle, avec ses $94 \%$ de jeunes scolarisés à dix sept ans et ses ouvriers de lrautomobile «bacheliers»; esquissée dans le rapport Les lycées et leurs études au seuil du XXI siècle de l'historien Antoine Prost sous la forme : «atteindre en 1993 lobjectif de 80\% de jeunes achevant une scolarité de second cycle», une politique et une formule , qui sera reprise par tous les gouvernements suivants, voient le jour : «80\% d’une classe d’âge au niveau du baccalauréat en l'an 2000». C’est dans ce contexte que, indissociable de lobjectif des $80 \%$, la loi de programme sur les enseignements technologiques et professionnels institue un nouveau diplôme, le baccalauréat professionnel (Prost in Moreau, 2002, p.95-111), attestant une aptitude à exercer « une activité professionnelle hautement qualifiée » tout en pouvant constituer un passeport pour l'enseignement supérieur. Le statut de ce diplôme s'avère en effet d’emblée ambigu, hésitant entre un débouché immédiat sur l`emploi et une accession de plein droit à lenseignement supérieur conférée 
par tout baccalauréat, hésitation entre perspective de qualification immédiate et perspective d'instruction prolongée. On peut voir dans cet exemple un concentré de traits significatifs : expression des incertitudes désormais caractéristiques du système éducatif français; glissement, pointé par Lucie Tanguy, de léducation à la formation, voire à la qualification, comme priorités ; mais aussi, déplacement des inégalités d'un rapport situé antérieurement entre extérieur et intérieur de l’enseignement secondaire à une ségrégation devenue désormais interne au système éducatif, entre filières de prestige et filières peu valorisées. Le sociologue Stéphane Beaud a ainsi pu montrer l'échec et la frustration de bacheliers professionnels confrontés à l'enseignement supérieur (Beaud, 2002).

Dans les années 1990, l'Etat a de plus en plus de velléités de se débarrasser de son caractère providentiel pour s’affirmer comme managérial, exerçant une gouvernance où - du fait d'une volonté politique affichée - sa part propre et celle de ses agents diminuent au profit de l'intervention d’acteurs privés, réputés plus libres et innovateurs, en référence au paradigme du libéralisme économique. Lécole et les politiques scolaires restent quant à elles hésitantes, soumises aux pressions managériales et libérales, tiraillées entre référence au principe marchand et au principe du bien commun, mais encore solidaires de ce dernier, c'est-à-dire de leurs fondamentaux historiques, notamment grâce à de puissantes réactions des personnels enseignants. Alors que l'obligation de résultats s'impose comme un nouveau credo sous la pression internationale des classements, du culte de la performance et de la révision générale des politiques publiques (RGPP), les projets réformateurs n’en continuent pas moins à être argumentés au nom de légalité des chances. La flexibilité des emplois, désormais soumis à concurrence sur un marché du travail devenu mondial, conduit à l'imposition du slogan de la formation tout au long de la vie, qui affecte la conception des études initiales et de la formation, ainsi que la manière de les évaluer (par compétences évolutives plus que par connaissances acquises).

La tendance libérale s’accentue au début des années 2000, et particulièrement après la présidentielle de 2007 à la faveur de la volonté renforcée de diminuer la régulation d'Etat et d'introduire des espaces concurrentiels dans le secteur public lui-même. C'est le néolibéralisme qui cherche alors à s'imposer ; celui-ci se présente à la fois comme une doctrine économique et comme une anthropologie, visant à promouvoir les valeurs du marché et de la prise de risque jusque dans les comportements humains. Si les ferments d'une telle anthropologie devaient être complètement mis en œuvre, ils pourraient donner lieu à un type de socialisation où, comme certains manuels de coaching le recommandent et comme le pratiquent certaines écoles de commerce, l’individu est invité à se penser sur le mode de l’entreprise (« entreprise de soi »). En matière éducative, « lécole néolibérale désigne un certain modèle scolaire qui considère léducation comme un bien essentiellement privé et dont la valeur est avant tout économique [...] elle est orientée [...] vers les objectifs de compétitivité qui prévalent dans léconomie globalisée » (Laval, 2003, p. 7 et 9). La réforme conduisant à une autonomie et à un autofinancement progressif des universités publiques dans un cadre concurrentiel mondial apparaît comme le laboratoire de cette politique. Conjuguée à la suppression des organismes de planification (2006), la volonté de suppression de la carte scolaire dans le second degré - remise en cause du principe gaulliste dorganisation stricte du système éducatif (cf. ci-dessus)- va dans le sens de la dérégulation, de la compétition entre les établissements, et de la concurrence entre les familles.

La récente réforme dite du " socle commun de connaissances et de compétences », qui prend place dans la loi dorientation et de programme (2005), peut être appréciée au regard de ces orientations. La présentant comme une véritable révolution copernicienne, ses partisans lui confèrent une valeur de plus grande portée dans la mesure où elle prétend concilier - pour les temps présents - instruction désormais pensée en termes de compétences fondamentales, socialisation (exprimée en termes de compétences sociales et civiques, d'autonomie et d'esprit d'initiative), et perspectives de qualification future (en ne raisonnant plus en termes de moyenne où les notes des différentes disciplines se compensent, mais en définissant le bagage absolument indispensable à l'exercice de toute activité professionnelle future au XXIe siècle, écho $\mathrm{du}$ « ce que nul ne peut ignorer » du XIXe). Les détracteurs dénoncent quant à eux le caractère réducteur du socle, fait de connaissances et de compétences minimum, en fait réservées aux plus démunis, et y voient une déclinaison néolibérale, distinguant instruction de très haut niveau pour quelques uns, promesse de qualification de même nature et d'insertion aisée dans la société, et instruction minimale, base d'une possible adaptation à la flexibilité des emplois, pour le grand nombre.

Envisagée dans sa globalité, la loi française de 2005 sur léducation offre des contrastes. En prétendant poursuivre des objectifs tels que $100 \%$ délèves détenteurs d'un diplôme, $80 \%$ au niveau bac, et $50 \%$ dans l'enseignement supérieur, elle rejoint des orientations de politique éducative initiées à léchelon international, et plus spécifiquement européen, s'inscrivant dans la visée d’une élévation générale du niveau des populations et dans celle du Life Long Learning, la relation école/économie faisant l'objet d’un développement tout particulier. Le rapport annexé affiche d’autres objectifs très volontaristes : la proportion de bacheliers généraux parmi les enfants de familles appartenant aux catégories socioprofessionnelles défavorisées devra augmenter de $20 \%$; la proportion de jeunes filles dans les séries scientifiques générales et technologiques de $20 \%$, etc. Il s'agit là d’autant de signes de ralliement de la politique scolaire de la France à une logique, européenne et mondiale, d’obligation de résultats, les systèmes éducatifs étant désormais pris dans une compétition et soumis à lévaluation internationale 
permanente de leurs performances. Un autre aspect transversal à la loi consiste dans l'introduction de lévaluation de la performance à tous les niveaux du système7, conformément à un nouveau paradigme budgétaire promu par la LOLF, loi organique relative à la loi de finances, relevant du concept de nouvelle gestion publique8 (Dardot, Laval, 2009, p. 377 et sq.). Le rapport annexé insiste : « Chaque année, un rapport annuel de performances, présenté à tous les niveaux d'organisation du service public, doit rendre compte de la mise en œuvre des orientations définies et de la réalisation des objectifs fixés par la présente loi pour la réussite de tous les élèves ". Cette logique relève d'une dimension systémique et concerne prioritairement le collectif, ensemble dans lequel les individus singuliers nont de valeur que rapportés aux classements et à la mesure des objectifs, confirmant la saisie d'une école incertaine d’elle-même par une vision économiste, hyper concurrentielle et « désindividualisante».

\section{Perspectives conclusives :}

Ainsi, des composantes de la trilogie au prisme de laquelle j’ai tenté de considérer l'évolution des institutions scolaires françaises, c'est (pour la masse des élèves) la qualification à courte vue et de faible niveau, voire peu monnayable sur le marché du travail, qui semble l’emporter, $\mathrm{du}$ fait de l'emprise d'une certaine conception économique sur l'école. Ce n’est pas que, dans mon esprit, les notions d'économie et de qualification soient haïssables en elles-mêmes; mais mes convictions humanistes m’inclinent à vouloir penser autrement l'idée de qualification en l'harmonisant avec celles d'instruction et de socialisation. Au début de la troisième période ci-dessus définie, un projet de réforme jamais abouti, connu sous le nom de plan Langevin-Wallon (rendu public en 1947), avait délimité les contours possibles d'une conciliation harmonieuse instruction/qualification/socialisation, à travers son slogan «former l'homme, le travailleur, le citoyen » (transmission de l'instruction et de la culture le plus longtemps possible dans un tronc commun à tous les élèves, préparation sérieuse à lemploi et qualification professionnelle, formation civique et socialisation citoyenne). Conscients de l'impossibilité de revenir en arrière, notre tâche déducateurs humanistes est, me semble-t-il, de penser à nouveaux frais une réforme inspirée des mêmes idéaux, appliquée aux conditions de l’environnement socio-économique contemporain, fondée sur la conviction que léducation et l'instruction étant des biens en soi, ces biens devraient être mis en œuvre dans le cadre d'une scolarité longue, cohérente, rendue véritablement « désirable » aux yeux des jeunes, et ne devraient en rien être présentés comme de possibles objets marchands, la conception marchande ayant pour effet totalement négatif de les soustraire à la considération prioritaire du bien commun et de l'intérêt général, références républicaines essentielles.

\section{Références bibliographiques}

Beaud, S. (2002). 80\% au bac ... et après ? Les enfants de la démocratisation scolaire. Paris : La Découverte,

Blais, M.-C., Gauchet, M. \& Ottavi, D. (2002). Pour une philosophie politique de l’éducation, Six questions d'aujourd'hui. Paris : Bayard.

Briand, J.-P. \& Chapoulie, J. M. (1992). Les collèges du peuple. Paris : INRP/CNRS.

Charlot, B. (1994) (dir.). Lécole et le territoire: nouveaux aspects, nouveaux enjeux. Paris: Armand Colin.

Dardot, P. \& Laval, C. (2009). La nouvelle raison du monde : essai sur la société néolibérale. Paris : La Découverte.

De Gaulle, C. (1970). Mémoires d’espoir, t. I, Le renouveau 1958-1962. Paris : Plon.

Derouet, J.-L. (1992). Ecole et justice. Paris : Métailié.

Dubet, F. \& Martuccelli, D. (1996). Théories de la socialisation et définitions sociologiques de lécole. Revue française de sociologie, XXXVII(4), 511-535.

Gauthier, R.-F. (2011). Malgoverno éducatif et questions curriculaires en France (thèse de doctorat). Université Lyon 2.

Goblot, E. (1925). La barrière et le niveau. Paris : Alcan. rééd. PUF, 2010.

Laval, C. (2003). L'école n'est pas une entreprise : le néolibéralisme à l’assaut de l'enseignement public. Paris : La Découverte,

Prost, A. (2002). La création du baccalauréat professionnel : histoire d’une décision. Dans G. Moreau (dir.), Les patrons, l'Etat, et la formation des jeunes. Paris : La Dispute.

Robert, A.-D. (2005). Les professeurs des petits lycées et leur représentation : crépuscule et postérité d’une idéologie catégorielle (1881-1965). Dans Caspard, P., Luc, J.-N. et P. Savoie (dir.), Lycées, lycéens, lycéennes, Deux siècles d'histoire (p. 317-329). Lyon : INRP.

Segré, M. \& Tanguy, L. (1970). Une nouvelle idéologie de lenseignement. VIIIéme congrès mondial de sociologie, Varna, 14-19 septembre, ronéoté.

7 En mai 2006, le service statistiques du ministère appelé DEP (division de lévaluation et de la prospective) est discrètement devenu DEPP (division de lévaluation, de la prospective et de la performance).

$8 \quad$ New public management, un des aspects de l'idéologie néolibérale. 
Seguy, J-Y. (2010). Les politiques de démocratisation de l'enseignement secondaire dans l'entre-deux-guerres ( thèse de doctorat). Université Lyon 2.

Spuller, E. (1892). L'éducation morale et civique de la démocratie, conférence à Châlon- sur-Saône, le 30.11.1884, in Education de la démocratie, Paris : Alcan.

Tanguy, L. (1986) (dir.). L'introuvable relation formation-emploi. Paris : La Documentation française.

Tanguy, L. (1991). L'enseignement professionnel en France. Des ouvriers aux techniciens. Paris : Presses universitaires de France.

Tanguy, L. (2011). Une histoire de la sociologie du travail en France : enquête sur le travail des sociologues 1950-1990. Paris : La Découverte. 